\title{
Publisher's Note: Distributed quantum dense coding with two receivers in noisy environments
} [Phys. Rev. A 92, 052330 (2015)]

Tamoghna Das, R. Prabhu, Aditi Sen(De), and Ujjwal Sen

(Received 30 November 2015; published 10 December 2015)

DOI: 10.1103/PhysRevA.92.069903

PACS number(s): 03.67.Hk, 03.67.Mn, 99.10.Fg

This paper was published online on 24 November 2015 with an error in the text on page 10. On page 10, the third line of the right-hand column should read as "opposite is true when the channels are noiseless. Here we see...." The paper has been corrected as of 1 December 2015. The text is correct in the printed version of the journal. 